\title{
Computer analysis of slope failure and landslide processes caused by water
}

\author{
I. Sarafis ${ }^{1} \&$ J. Zezulak ${ }^{2}$ \\ ${ }^{1}$ Mechanical Engineering Dept., T E I, Kavala, Greece \\ ${ }^{2}$ Dept. of Constructions, Czech University of Agriculture, Prague, \\ Czech Republic
}

\begin{abstract}
At headwater channels in particular, the landslide of slopes with water body intersection, together with earth dam failures frequently become a main contribution to debris flow. The use of a computer model for slope stability evaluation of an arbitrary earthwork based on the Bishop's method is discussed. Similarly to that of Peterson, it adopts the assumption of a circular shape of the sliding body and evaluates the balance between the forces acting in the direction of the failure (destructing forces) and the resisting forces. In contrast to Peterson, the Bishop's method considers solely the 'mobilised' shear stress as the only part of total shear stress over the sliding circle. The program applies both for earth dams and for natural land-sliding. The AutoCAD engine provides for visual generation of an almost unlimited number of shapes of the body 'segments', being interfaced with the input/output utilities of the soil mechanics module. Keywords: earth bodies, landslides affected by water, slip circle, CAD support.
\end{abstract}

\section{Introduction}

Apart from methods of finite elements and/or differences, the traditional slope stability solvers are classified into two categories, using either principles of elastic theory or conditions of boundary plastic balances. At the latter, the limiting state of sliding body is assumed and conditions of its genesis are examined. The correct presumption of the form and position of slipway surface is a crucial point of such analysis. Different authors consider the problem differently.

Possibility of an easy introduction of various types of soils into the earthwork and introducing features of non-homogeneity into its structure becomes main 
advantage of the slip-circle oriented methods. Most often assumed cylindrical, though composition of several planar elements, wedge-shaped or entire block soil sliding can also simulate processes in a slipping zone. One can consider the form of lemniscates, logarithmic curves, spirals but the circles still prevail. An experience has proven that, no matter how large the radius could be, the presumption of circular cylindrical surface fits well both to forming the failure and to simplicity of algorithmic structure. This is why it still prevails in many methods of slope stability determination such as the method of Peterson and, more recently that of Bishop [3]. A reader with deeper interest in theoretical basis of the algorithm is kindly referred to the program manual, Novotny [1], Zezulak and Novotny [2].

\section{Mathematical formulation and algorithmic structure}

\subsection{Bishop's method: principles}

After Bishop, solely the mobilized portion of the shear stress is introduced into inactive forces' calculation, as the only part of its full value,

$$
\tau_{f}=\frac{1}{F}\left[c^{\prime}+(\sigma-u) \operatorname{tg} \varphi^{\prime}\right]
$$

$$
\begin{aligned}
& \text { where } \tau_{f} \quad \text { shear stress } \\
& F \quad \text { resulting safety factor } \\
& c^{\prime} \quad \text { effective soil cohesion along the slip circle } \\
& \varphi \quad \text { effective angle of friction along the slip circle } \\
& \sigma \quad \text { normal tension actuating upon slip surface }
\end{aligned}
$$

As the safety factor is not known beforehand its evaluation requires an iterative procedure. We write an equilibrium condition at the centre of an investigated slip circle. For the value of $\sigma$ we introduce $\sigma=P / l$. For active and passive moments with respect to the centre of a circle it follows, Figure 1:

$$
\begin{aligned}
& M_{a}=\sum W x \\
& M_{b}=R \sum\left(c^{\prime} l+P^{\prime} \operatorname{tg} \varphi^{\prime}\right)
\end{aligned}
$$

If considering the differences in forces $E_{i}, E_{i+1}$ and $X_{i}, X_{i+1}$ then the force $P$ ' can be evaluated from cumulative condition written in vertical direction, Figure 1:

$$
P^{\prime}=\frac{W+X_{i}-X_{i+1}-l\left(u \cos \alpha+\frac{c^{\prime}}{F} \sin \alpha\right)}{\cos \alpha+\frac{\operatorname{tg} \varphi^{\prime} \sin \alpha}{F}}
$$

In view of further analysis let now introduce $X$-R $\sin \alpha$, hence $R=X / \sin \alpha$. The coefficient of Bishop $\bar{B}$ follows from

$$
u=\bar{B}\left(\frac{W}{b}\right)
$$




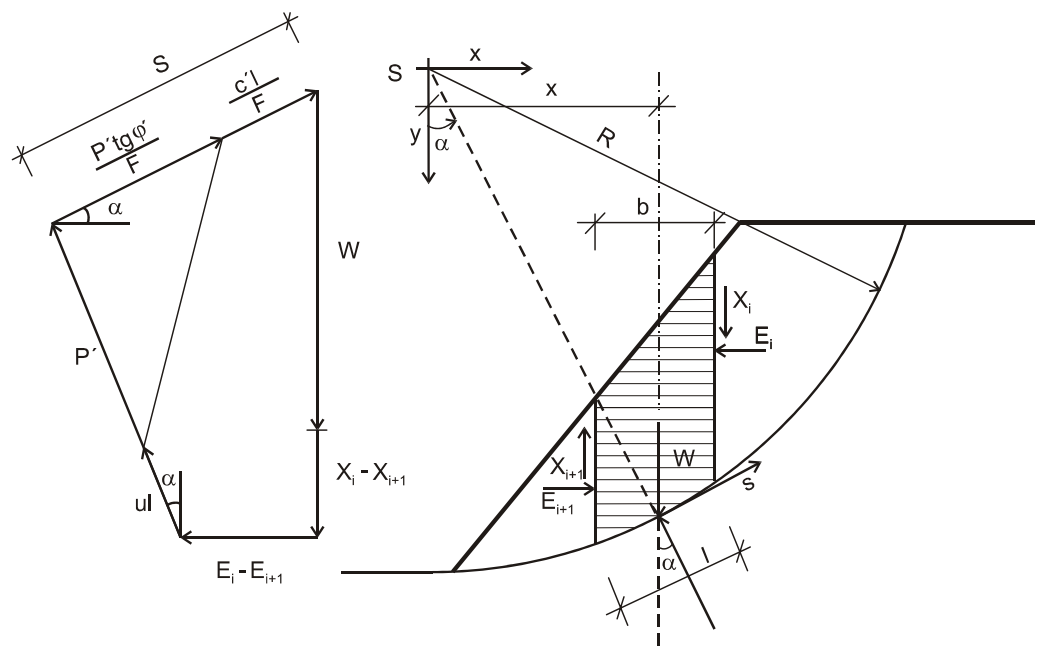

Figure 1: Equilibrium of active and passive forces.

It is called a global pore pressure coefficient, defined as

$$
\bar{B}=\frac{\Delta u}{\Delta \sigma}=\frac{\text { pore pressure increment }}{\text { max tension increment }}
$$

The $\bar{B}$ coefficient should be laboratory-determined for load reflecting the reallife conditions. We express the safety degree as a rate of passive and active moments. After substitution of the above expression (3) for $P^{\prime}$, we receive, after rearranging the original implicit expression for the safety degree $F$ after Bishop,

$$
\begin{aligned}
& F=\frac{M_{a}}{M_{b}}=\frac{R \sum\left(c^{\prime} l+P^{\prime} \operatorname{tg} \varphi^{\prime}\right)}{\sum W x}= \\
& =\frac{1}{\sum W \sin \alpha} \sum\left[c^{\prime} b+\operatorname{tg} \varphi^{\prime}\left(W(1-\bar{B})+X_{i}-X_{i+1}\right)\right] \frac{1}{\cos \alpha+\frac{\operatorname{tg} \varphi^{\prime} \sin \alpha}{F}}
\end{aligned}
$$

According to Taylor [4], and in spite the fact that forces at boundaries of elementary strips do not vanish, the overall forces' equilibrium is preserved. One can suppose $\Sigma\left(X_{i}-X_{i-1}\right)=0$ with sufficient accuracy. Then the eqn. (6) can be further simplified.

\subsection{Pore pressure analysis}

In water saturated sector of the earth-body the pore pressure can be evaluated by introduction of the factor $r_{u}$ into governing equation,

$$
r_{u}=u / w^{\times}
$$

where $r_{u} \quad$ dimensionless factor

$u \quad$ neutral tension (equal to hydrostatic pressure)

$w^{x} \quad$ tension from weight overburden considered at neutral tension pressure centroid 
The distribution of the factor $r_{u}$ follows from orthogonal flow net. From forces' balance the following can be worked out, in analogy to its original (6),

$$
F=\frac{1}{\sum W \sin \alpha} \sum\left[c^{\prime} b+W\left(1-r_{u}\right) \operatorname{tg} \varphi^{\prime}\right] \frac{1}{\cos \alpha+\frac{\operatorname{tg} \varphi^{\prime} \sin \alpha}{F}}
$$

\subsection{Governing equation algorithmic structure}

Here we start again from the eqn. (6) that will be adopted into an algorithmic form necessary to accomplish: arbitrarily torn up ground, non-homogenous structure of the earth body cross section, arbitrary composition of soils of different properties, introduction of hydrodynamic pressure caused by seepage water, slope stability affected by earthquake and, external load upon the crest.

\subsubsection{Broken surface and non-homogenous structure of the earth body}

If the soils within various segments are homogenous, for each elementary strip $d x$ it applies $d W=\gamma_{z} h(x) d x$ provided the function $h(x)$ of the strip height is smooth and continuous.

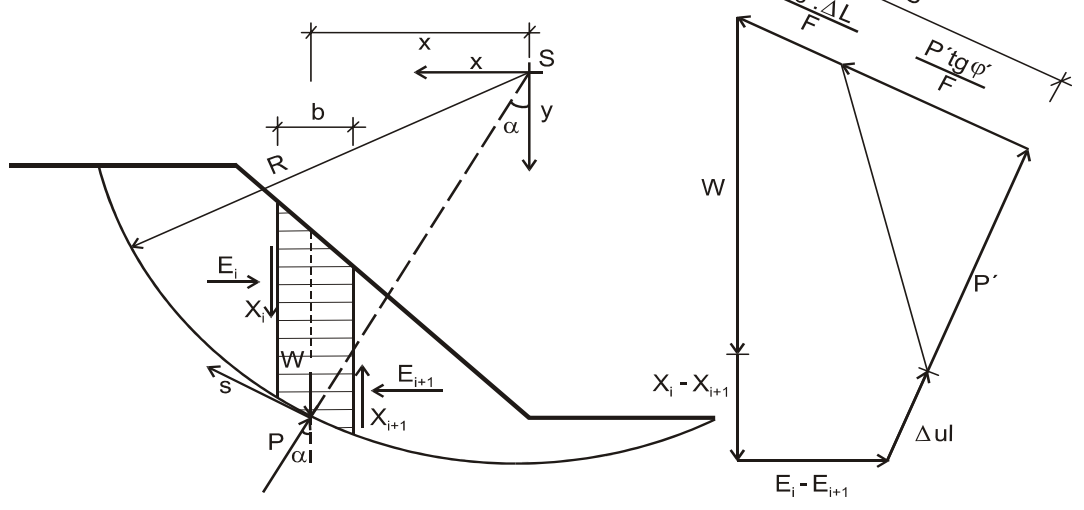

Figure 2: Equilibrium of active and passive forces, pore pressure included.

The $\gamma_{z}$ is a specific weight of the $z$-indexed soil. The elementary strips $d h$ can be integrated throughout entire region $\Gamma$ of the cross section. After rearrangement, the safety factor is computed with the equation, Figure 3:

$$
F \frac{\sum_{\Gamma} \int_{x_{i}}^{x_{j}}\left[c^{\prime}+\operatorname{tg} \varphi^{\prime} \gamma_{\mathrm{z}} h(x)(1-\bar{B})\right] \frac{1}{\cos \alpha+\frac{\operatorname{tg} \varphi^{\prime} \sin \alpha}{F}} d x}{\sum_{\Gamma} \int_{x_{i}}^{x_{j}}\left[c^{\prime}+\gamma_{\mathrm{z}} h(x) \sin \alpha\right] d x}
$$

Non-homogenous earthworks can develop into quite complex formation and usually require $\mathrm{CAD}$ support for its visual definition. Here we apply the 
following procedure in computerization: (1) first we specify types of soil that differ in physical-mechanical properties and introduce co-ordinate system so that its origin coincides with the centre of the slip circle, (2) we transform all the geometric and soil layer data hereinto, (3) for sake of numerical integration we subdivide the segment above considered surface into elementary pinstripes.

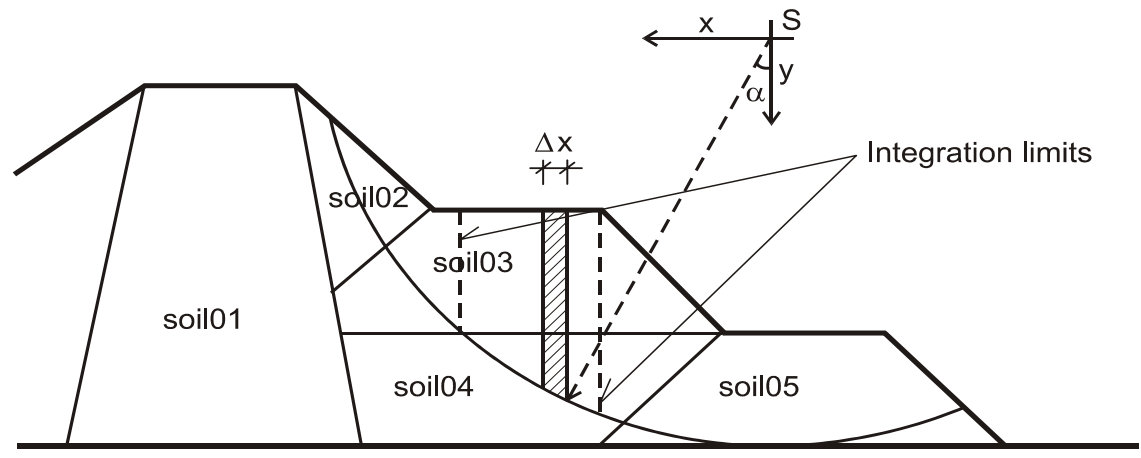

Figure 3: Definition sketch of non-homogenous earth dam.

Any two breaking points on earthwork surface, soil transitions and/or slip circle intersect define a limit of integration, see Figure 4: for more details. We arrive to interim form of governing equation, yet missing the impact of earthquake,

$$
\begin{aligned}
& F=\frac{\sum_{\Gamma} \int_{x_{i}}^{x_{j}}\left[c^{\prime}+\operatorname{tg} \varphi^{\prime} \gamma_{\mathrm{z}} \Delta W(1-\bar{B})\right] \frac{F R}{F \sqrt{R^{2}-x^{2}}+\operatorname{tg} \varphi^{\prime} x} d x}{\sum_{\Gamma} \int_{x_{i}}^{x_{j}} \frac{x}{R}(\Delta W) d x} \\
& \Delta W=\left[A_{R}+B_{R}+\left(D_{R}-C_{R}\right)\left(x_{i}-x\right)+\gamma_{1} \sqrt{R^{2}-x^{2}}\right]
\end{aligned}
$$

where $A_{R}, B_{R}, C_{R}$ and $D_{R}$ are auxiliary coefficients [2].

\subsubsection{Earthquake impacted slope stability}

In earthquake prone regions particular attention should be given to the slope stability. Provided its impact is proportional to the soil specific weight and it acts horizontally, it can be understood as an internal body force, $Z=\xi \gamma_{z}$, where $\xi$ is a coefficient proportional to the quake intensity,

$$
F=\frac{\sum_{\Gamma} \int_{x_{i}}^{x_{j}}\left[c^{\prime}+\operatorname{tg} \varphi^{\prime} \gamma_{z} \Delta W\left(1-\xi \frac{x}{R}\right)-u\right] \frac{F R}{F \sqrt{R^{2}-x^{2}}+\operatorname{tg} \varphi^{\prime} x} d x}{\sum_{\Gamma} \int_{x_{i}}^{x_{j}}(\Delta W)\left(\frac{x}{R}+\xi \frac{\sqrt{R^{2}-x^{2}}}{R}\right) d x}
$$




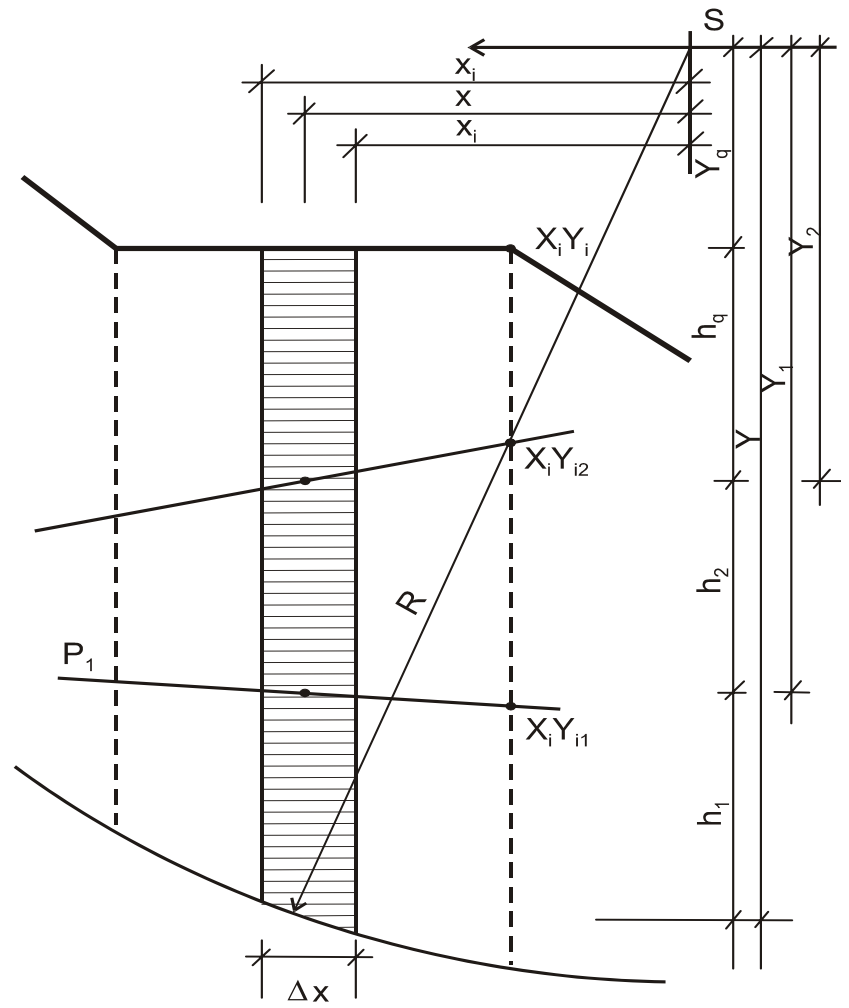

Figure 4: Elementary strip weight definition.

\subsubsection{Complete formulation of the governing equation}

If compared with original eqn. (6) of Bishop, the eqn. (11) conforms with all additional impacts, such as arbitrary form of ground, non-homogenous structure of the earth body, arbitrary composition of soils of different properties, introduction of hydrodynamic pressure caused by seepage water, slope stability affected by earthquake and external load over the crest.

\subsubsection{Field of application}

The program solves stability of an arbitrary geometry of earth body for maximum of 14 types of soils in the construction/natural hill slope. The number of geometry-definition lines (called the 'segments') is unlimited. The program works in a man-machine conversation control and user-defined data files. The import/export of the data from/into a CAD system is supported.

The program is implemented within design offices throughout the Czech Republic and also in several UN projects (Bangladesh, Burma). All the data processing and the draftsmen work should be preferably CAD computerized. On the output the program provides for the critical slip-circle definition and corresponding minimum safety degree. Its postprocessor can also evaluate total bulk of soil entering the stream. 


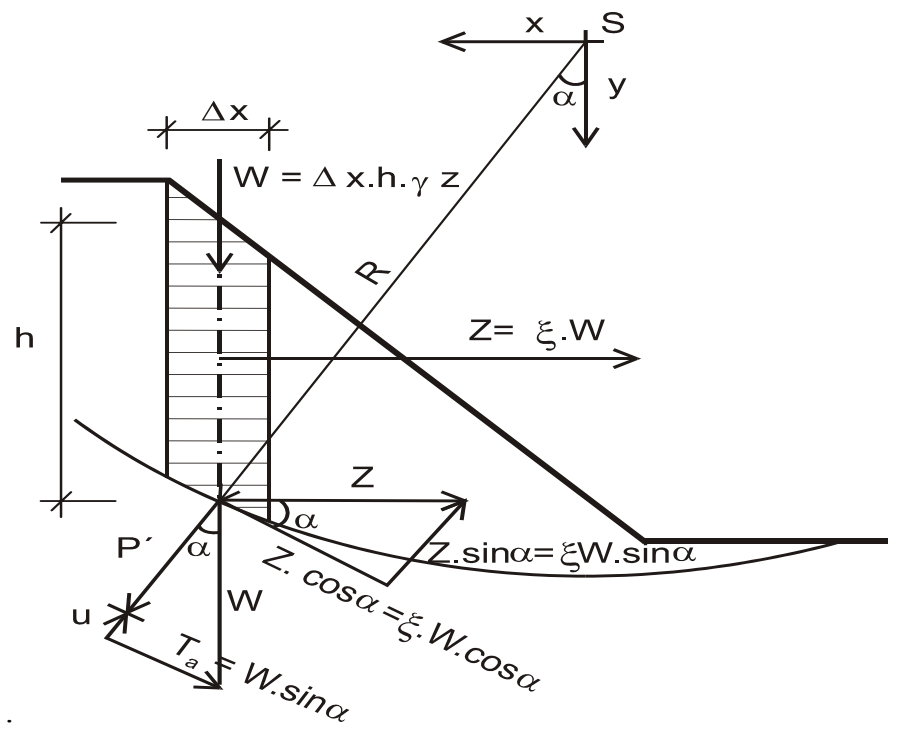

Figure 5: Resolution of forces impacted by earthquake.

\subsubsection{Program requirements}

(1) Given geometry of the earthwork and distribution of individual soil types within the earth body. The Cartesian coordinate system $x$-horizontal, $y$-vertical.

(2) Pre-processed (or estimated) phreatic surface of groundwater table creates boundary between the dry and the wet soil.

(3) The following soil parameters in all segments of the construction:

-Specific weight of water $\left(\gamma\right.$-water) $\left(\mathrm{kN} . \mathrm{m}^{-3}\right)$.

-Specific weight of individual soil types $\left(\gamma_{z}\right.$-soil $)\left(\mathrm{kN} \cdot \mathrm{m}^{-3}\right)$.

-Angles of internal friction of the soils $\varphi^{\prime}$.

- $\quad$-Cohesion factors of the soils $c^{\prime}\left(\mathrm{kN} \cdot \mathrm{m}^{-3}\right)$.

-Bishop's coefficients.

(4) Constraint for computation process in form of a boundary polyline, beyond which no circles can be generated.

(5) Value of external load acting upon the construction, if any.

\subsubsection{Selection of computational strategy}

The program Soil_SlipCircle provides for three options in minimum safety factor analysis:

(1) Single circle: given fixed centre and radius $R$.

(2) Set of circles: given fixed centre and increment in $R$.

(3) Set of circles: given initial centre, increments in $R$, and in $x$ and $y$ coordinates.

The strategies vary with respect to complexity of calculations. In the first case only a single slip circle is computed while given its centre and radius. The second case provides a set of circles from a given centre and increment in the 
radius. The third case creates all possible circles and it is a most effecting option in defining the safety factor. The program terminates the search when solution circle hits constraining boundary.

\subsubsection{Input data}

(1) Earthwork geometry: SegmNo, $x_{\text {start }}, y_{\text {start }}, x_{\text {end }}, y_{\text {end }}$, SoilNo, ExtLoad available for each geometry-construction line/polyline of the earthwork. The text-form data can be replaced by DXF entry using AutoCAD.

(2) Soil parameters: FileHeader, JobDescriptor, No of the $1^{\text {st }}$ wetted soil from the top, Earthquake coefficient (RichterScale), SoilNo and related parameters (specific weight $\gamma_{z}$, internal friction angle $\varphi$ ', soil cohesion factor $c$ ', Bishop's coefficient $B$ ).

(3) Man-machine conversation: choice of geometry data input from the text file or from the DXF-file (e.g. from AutoCAD), selection of the computational strategy ( 3 options). The program controls the syntax of entered data and issues necessary warnings.

\subsubsection{Output data}

(1) Text output: program generates the detailed review of input data: soil parameters, strategies used in search for the minimum safety factor, starting conditions included and results of the analysis: list of all circles when safety factor $F<3.0$, minimum safety factor $F$ found, statistics of the results (number of circles computed, elapsed computer time, etc.).

(2) Visual output: the earthwork geometry and a critical slip circle can be viewed by any CAD system with DXF interface.

\subsection{Selected examples and case studies}

Two examples are provided to demonstrate the Soil_SlipCircle functioning for non-homogenous earth dams stability evaluation:

(1) Primitive case: soils layered horizontally.

(2) Complex case: soils layered with respect to dam building technology.

\subsubsection{Example of the dam, soils deposited horizontally}

This is a simple case to demonstrate virtue of the geometry definition using the CAD system. The earthwork consists of 5 horizontally layered soils of various physical-mechanical properties. Computational strategy is set to option 2, see 2.3.6, i.e. set of circles is given of fixed centre and increment in $R$, see Figure 6 :

\subsubsection{Soils deposited with respect to the dam building technology}

The case study of the dust and soot-collecting reservoir reflects the technology of gradual filling of the pool by thermal power plant soot and dust. Although obsolete, it may serve an example for natural unstable hill slopes stability analysis. The case consists of 115 lines created in AutoCAD, and of 12 different soil categories. The program has evaluated more than 1600 events, to achieve the value of minimum safety degree $F=1.694$, Figure 7 : 


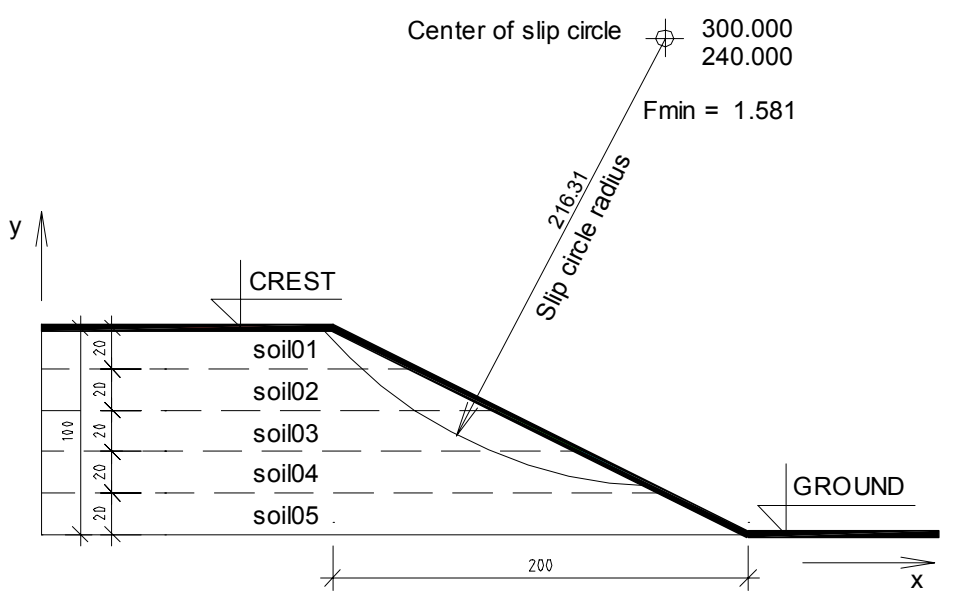

Figure 6: Example of 5 horizontally layered soils.

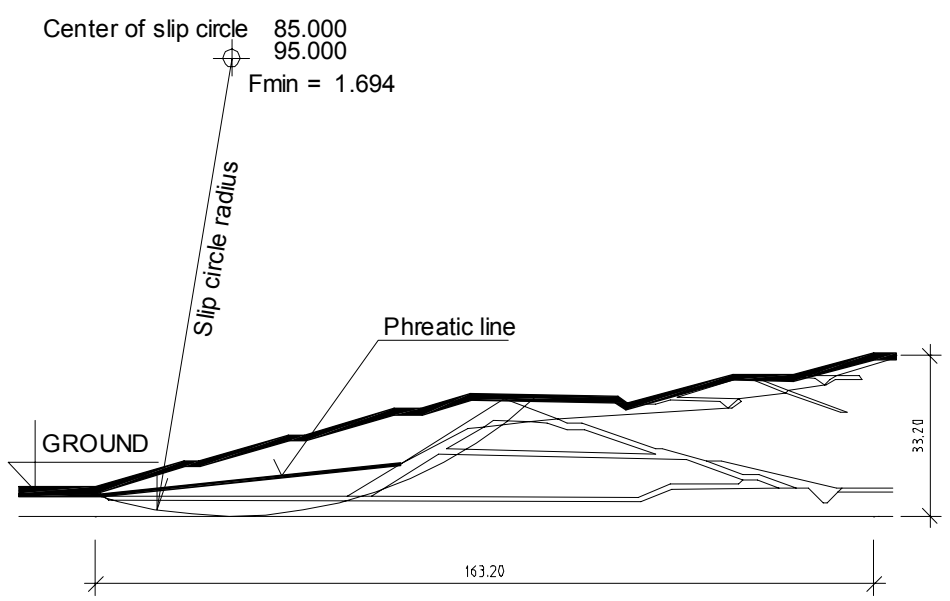

Figure 7: Example of complex earthwork system.

\section{Conclusions}

The program Soil_SlipCircle evaluates the slope stability of non-homogeneous earth bodies by modified method of Bishop. Its main features are as follows:

(1) The slip circles are generated in three program options:

(a) Given the radius and the center.

(b) Given the center and increment of the radius.

(c) Given the set of centers, increments in $\mathrm{X}, \mathrm{Y}$ and in the radius. 
(2) Construction of the constraining polyline that prevents circle abnormal generation.

(3) Inconsistency checking of geometry data on input.

(4) Introduction of earthquake- and Bishop's coefficients and of external load upon the ground.

(5) Two options in the soil water saturation: dry soil, phreatic surface

\section{References}

[1] Novotny, R., Program Bishop, Slope stability evaluation (in Czech), Energoprojekt Praha, Ltd., 1992.

[2] Zezulak, J., Novotny, R., Program Soil_SlipCircle, Theory and Users' Guide, Czech Univ. of Agricult. Dept. of Constructions, Prague, 2006.

[3] Bishop, A. W., Morgenstern, N., Stability Coefficients for Earth Slopes, Geotechnique X 4 pp.129-150.

[4] Taylor, D., W., Fundamentals of Soil Mechanics, New York, 1948.

[5] Mencl, V., Soil and rock mechanics (in Czech), Academia Praha, 1966.

[6] Bazant, Z., Foundation Engineering (in Czech), Academia Praha, 1973. 